\title{
High-Efficiency Metasurfaces with $2 \pi$ Phase Control Based on Aperiodic Dielectric Nanoarrays
}

\author{
Sihui Shang ${ }^{1,2, \dagger}$, Feng Tang ${ }^{2,+}$, Xin $\mathrm{Ye}^{2}$, Qingzhi $\mathrm{Li}^{2}{ }^{2}$, Hailiang $\mathrm{Li}^{3, *}$, Jingjun $\mathrm{Wu}^{2}$, Yiman $\mathrm{Wu}^{2}$, \\ Jun Chen ${ }^{2}$, Zhihong Zhang ${ }^{1, *}$, Yuanjie Yang ${ }^{1}$ and Wanguo Zheng ${ }^{2, *}$ \\ 1 School of Physics, University of Electronic Science and Technology of China, Chengdu 610054, China; \\ 201721040131@std.uestc.edu.cn (S.S.); dr.yang2003@uestc.edu.cn (Y.Y.) \\ 2 Research Center of Laser Fusion, China Academy of Engineering Physics, Mianyang 621900, Sichuan, China; \\ tangfengf3@126.com (F.T.); yexin@caep.cn (X.Y.); dearlqz@163.com (Q.L.); jingjunwu163@163.com (J.W.); \\ manmanna@yeah.net (Y.W.); chenjun19950110@163.com (J.C.) \\ 3 Key Laboratory of Microelectronic Devices \& Integrated Technology, Institute of Microelectronics of Chinese \\ Academy of Sciences, Beijing 100029, China \\ * Correspondence: lihailiang@ime.ac.cn (H.L.); zhihongzhang@uestc.edu.cn (Z.Z.); group_ye@163.com (W.Z.); \\ Tel.: +86-1348-867-5143 (H.L.); +86-1360-817-4673(Z.Z.); +86-1539-9778-0786 (W.Z.) \\ + These authors contribute equally to this article.
}

Received: 17 December 2019; Accepted: 27 January 2020; Published: 31 January 2020

check for updates

\begin{abstract}
In this study, the high-efficiency phase control Si metasurfaces are investigated based on aperiodic nanoarrays unlike widely-used period structures, the aperiodicity of which providing additional freedom to improve metasurfaces' performance. Firstly, the phase control mechanism of Huygens nanoblocks is demonstrated, particularly the internal electromagnetic resonances and the manipulation of effective electrical/magnetic polarizabilities. Then, a group of high-transmission $\mathrm{Si}$ nanoblocks with $2 \pi$ phase control is sought by sweeping the geometrical parameters. Finally, several metasurfaces, such as grating and parabolic lens, are numerically realized by the nanostructures with high efficiency. The conversion efficiency of the grating reaches $80 \%$, and the focusing conversion efficiency of the metalens is $99.3 \%$. The results show that the high-efficiency phase control metasurfaces can be realized based on aperiodic nanoarrays, i.e., additional design freedom.
\end{abstract}

Keywords: metasuface; Huygens; granting; metalens; phase control

\section{Introduction}

Due to the restricted permittivity and permeability, natural materials cannot manipulate electromagnetic waves arbitrarily and wilfully. Electromagnetic properties of metamaterials can be designed artificially, breaking the limit of natural materials and having great potentials in the applications of imaging [1,2], solar cells [3], holographic [4,5], etc. However, the disadvantages of high loss, fabrication challenges and strong dispersion [6] limit the possible applications of 3D metamaterial, resulting in the appearance of metasurfaces, i.e., sub-wavelength nanostructure (meta-atom) arrays. Metasurfaces can compress the thickness of traditional optical elements [7] to the sub-wavelength level, performing an arbitrary manipulation of amplitude [8-11], phase [12-16], and polarization [17-19]. Unlike plasmonic ones [20-24], dielectric metasurfaces [25] have made significant breakthroughs in optical refractive index sensors [26], biochemical sensing devices [27], and microwave sensors [28], particularly based on semiconductor materials like Si, have lower loss and higher efficiency [29], and compatibility with the CMOS facility.

Electromagnetic fields can excite both electric and magnetic dipoles in dielectric nanostructures $[29,30]$. It is shown that the simultaneous manipulation of electric and magnetic 
dipoles can achieve $2 \pi$ phase control in a single-layer dielectric nanoarray. This kind of metasurface has a theoretical transmittance approaching 100\%, i.e., Huygens source [29,31-33]. Recently, some reports have demonstrated that Huygens metasurface [29,31,34], using $\mathrm{Si}$ and $\mathrm{TiO}_{2}$ [35] as low-loss semiconductor materials, can realize high efficiency in $2 \pi$ phase wavefront control. Metamaterials are expected to integrate with on-chip nanophotonic devices, and a variety of functional materials incorporated to reconfigurable or tunable metasurfaces is also presented [36,37]. Moreover, many studies have shown that MEMS tunable dielectric metasurfaces lenses pave the way for MEMS-integrated metasurfaces, which can be fully used as a platform for tunable and reconfigurable optical [38,39]. Existing studies on metasurfaces are mainly based on periodic nanoarrays of meta-atoms $[14,17,30]$, of which the optical properties will deviate from the designed values when constructing metasurfaces, finally causing the undesired optical performance and low efficiency of metasurfaces.

The $830 \mathrm{~nm}$ wavelength laser is widely used in clinical and medical applications [40-42]. In this study, a method of constructing Huygens metasurfaces based on aperiodic Si nanoarrays is proposed to approach phase manipulation of $830 \mathrm{~nm}$ light, in which the edge to edge distance keeps constant. This approach can provide additional design freedom and improve metasurfaces' performance. The studied metasurfaces can replace traditional gratings and lenses to conduct optical deflection, focusing, imaging, etc. Firstly, the optical properties of Si meta-atoms are studied by finite-difference time-domain (FDTD) simulations and the internal phase control mechanism is demonstrated based on the coupling of electric/magnetic dipoles. Then, a group of $\mathrm{Si}$ meta-atoms with $2 \pi$ phase control is sought by sweeping the geometrical parameters. Finally, two example metasurfaces of grating and parabolic lens are constructed and analyzed numerically in the near-infrared range. The results show that the high-efficiency phase control metasurfaces can be realized based on aperiodic nanoarrays.

\section{Design of Meta-atoms and Methods}

All simulations in this study were implemented using FDTD Solutions, a commercial software package provided by Lumerical Solutions, Inc. The simulation region of meta-atoms was surrounded with periodic boundaries in the $\mathrm{x}$-axis and $\mathrm{y}$-axis directions and perfectly matched layers in the $\mathrm{z}$-axis direction. The size of the nanoblocks was set from $180 \mathrm{~nm}$ to $430 \mathrm{~nm}$. The height of nanoblocks was set at 132nm. The size effect on transmittance and phase was calculated. All objects, sources (S parameter), and monitors were laid in this simulation region. The PML boundary was used to the $x$-axis, $y$-axis, and z-axis for simulating optical devices. The TFSF (total-field scattered-field) source, all objects, and monitors were limited in the simulation region. The refractive indexes of silicon $(\mathrm{Si})$ and glass $\left(\mathrm{SiO}_{2}\right)$ come directly from the database of the FDTD software.

According to the analytical model of Huygens meta-atoms, the transmittance and reflection coefficients [29] can be expressed as below:

$$
\begin{gathered}
t=1+\frac{2 i \cdot \gamma_{e} \omega}{\omega_{e}^{2}-\omega^{2}-2 i \gamma_{e} \omega}+\frac{2 i \cdot \gamma_{m} \omega}{\omega_{m}^{2}-\omega^{2}-2 i \gamma_{m} \omega} \\
r=\frac{2 i \cdot \gamma_{e} \omega}{\omega_{e}^{2}-\omega^{2}-2 i \gamma_{e} \omega}-\frac{2 i \cdot \gamma_{m} \omega}{\omega_{m}^{2}-\omega^{2}-2 i \gamma_{m} \omega}
\end{gathered}
$$

where $\omega_{e}$ and $\omega_{m}$ represent the resonant frequencies of the electric dipole and the magnetic dipole, respectively. $\gamma_{e}$ and $\gamma_{m}$ represents the damping coefficient of the electric/magnetic dipole. When $\omega=\omega_{e}=\omega_{m}$ and $r=0$, the individual non-reflective nanoblock can be regarded as an ideal Huygens source. Metasurfaces are composed of nanoblocks (i.e., meta-atom) with different sizes, arrangements, etc. Thus, the array of meta-atoms with the same parameters is investigated firstly, as shown in Figure 1. An array of nanoblocks, with height $\mathrm{H}=132 \mathrm{~nm}$, width $\mathrm{W}=370 \mathrm{~nm}$, length $\mathrm{L}=330 \mathrm{~nm}$, and the period $\mathrm{P}=460 \mathrm{~nm}$, is on top of silica substrate. The incidence is assumed to be a plane wave, propagating along the $\mathrm{z}$-axis and being polarized along the x-axis. For light propagating through the array, the 
transmittance spectrum has two dips caused by the magnetic $\left(\lambda_{\mathrm{m}}=827 \mathrm{~nm}\right)$ and electric $\left(\lambda_{\mathrm{e}}=910 \mathrm{~nm}\right)$ resonances. The corresponding electromagnetic distributions are presented in Figure $1 \mathrm{c}-\mathrm{f}$, showing the patterns of magnetic/electric dipoles. When the two dipoles overlap via changing the nanoblocks' geometrical parameters, $100 \%$ transmission can be realized theoretically.

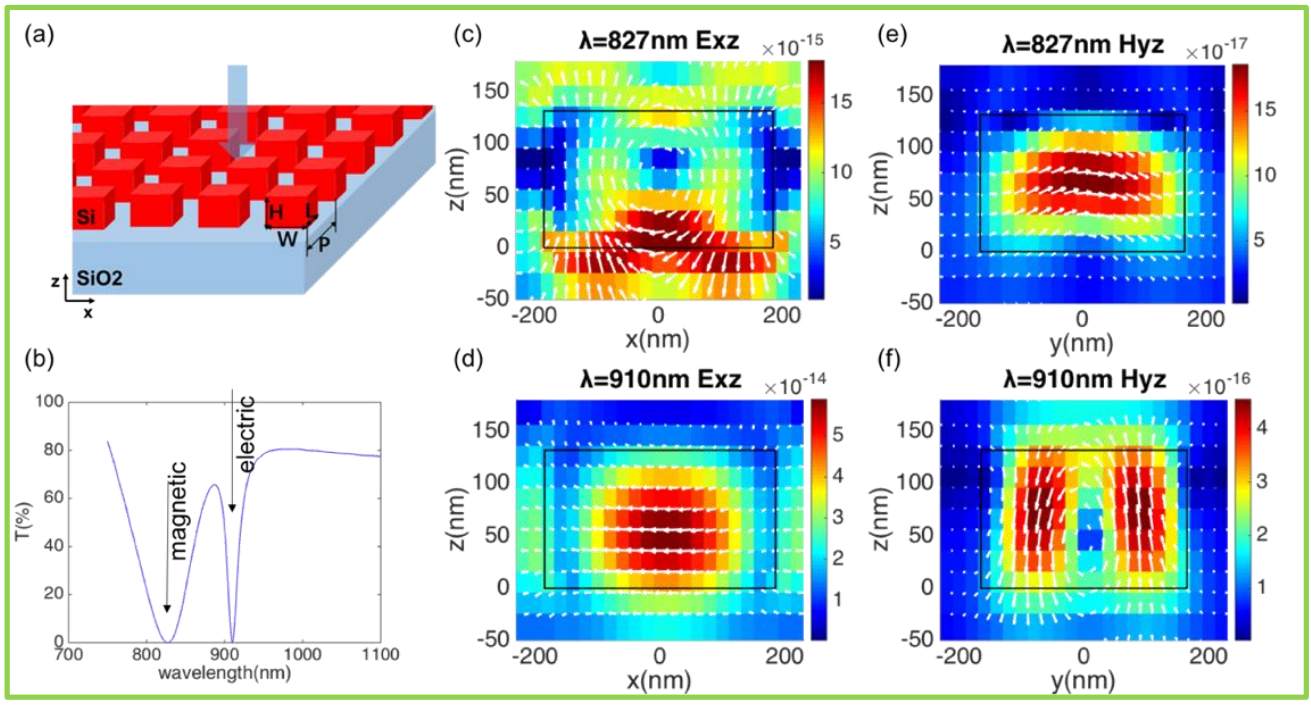

Figure 1. Schematic of meta-atoms and internal electromagnetic excitation. (a) Three-dimensional view of meta-atoms, i.e., a nanoblock array on top of the silica substrate. Nanoblocks' height $\mathrm{H}=$ $132 \mathrm{~nm}$, width $\mathrm{W}=370 \mathrm{~nm}$, length $\mathrm{L}=330 \mathrm{~nm}$, and period $\mathrm{P}=460 \mathrm{~nm}$. (b) Transmittance spectrum of meta-atoms with two dips for magnetic $\left(\lambda_{m}=827 \mathrm{~nm}\right)$ and electric $\left(\lambda_{e}=910 \mathrm{~nm}\right)$ resonances. $(\mathbf{c}, \mathbf{d})$ Electric/magnetic field distribution of the magnetic dipole, which is indicated by the vortex-like electric pattern, on two orthogonal cross-sections of the nanoblocks. (e,f) Electric/magnetic field distribution of the electric dipole, which is indicated by the vortex-like magnetic pattern, on two orthogonal cross-sections of the nanoblocks. Electric and magnetic field amplitudes are normalized to their maximum values in the study.

By tuning $\omega_{e}, \omega_{m}, \gamma_{e}$ and $\gamma_{m}$ via blocks' sizes, $r$ can be 0 , i.e., the magnetic/electric dipoles overlap and the impedance matches with the surrounding medium. In the case, the transmission coefficient $t$ is $100 \%$, where a non-reflective Huygens source appears. Tuning the nanoblocks in the vicinity of the parameters at the match point, the phase of $t$ can cover the $0-2 \pi$ range and the transmittance is still near $100 \%$. Transmittance and phase variation of light are calculated as a function of nanoblocks' width $W$ and length $L$ with high $\mathrm{H}$ and period $\mathrm{P}$ are parameters that need to be optimized, as shown in Figure 2. To avoid the deviation of optical properties in constructing metasurfaces, the edge to edge distance $D$ is kept constant when tuning the nanoblocks. The highest transmittance achieved by the nanoblocks in the $0-2 \pi$ full-phase space is shown in Figure 2c. Eight points marked by stars are selected to construct metasurfaces. The transmittance and phase of the selected meta-atoms with size errors are shown in Figure A2.

The optical properties of meta-atoms are controlled by manipulating the charge oscillation with nanoboundaries. The effective optical properties are different from the bulk. The effective electrode rate $\alpha_{e}^{e f f}$ and the effective magnetic polarizability $\alpha_{m}^{e f f}$ are respectively expressed [31] as:

$$
\begin{gathered}
j \omega \alpha_{e}^{e f f}=\frac{2 *(1-t-r)}{\sqrt{\mu / \varepsilon}(1+t+r)} \\
j \omega \alpha_{m}^{e f f}=\frac{2 * \sqrt{\mu / \varepsilon}(1-t+r)}{(1+t-r)}
\end{gathered}
$$


Here, $\omega$ is an angular frequency, $\varepsilon$ and $\mu$ represent a dielectric constant and magnetic permeability in a vacuum, and $t$ and $r$ are transmission and reflection coefficients, respectively.

The effective electric/magnetic polarizability of the selected eight meta-atoms in Figure 2 are shown in Figure 3. The solid red lines indicate the real part of the ideal effective polarizability when the transmittance is $100 \%$ while the blue one is the imaginary part. The red circles indicate the real part of the actual effective polarizability of the selected meta-atoms while the blue diamonds are the imaginary part. Moreover, we find that the real part of the meta-atomic polarizability designed in this paper is basically consistent with the ideal values, and there is a small amount of deviation in the imaginary part, which may be related to the absorption inside the meta-atoms. We calculated our $\mathrm{Q}$ factors and dephasing times of all the electromagnetic resonances of the Si nanoblocks, as shown in Figure A3.
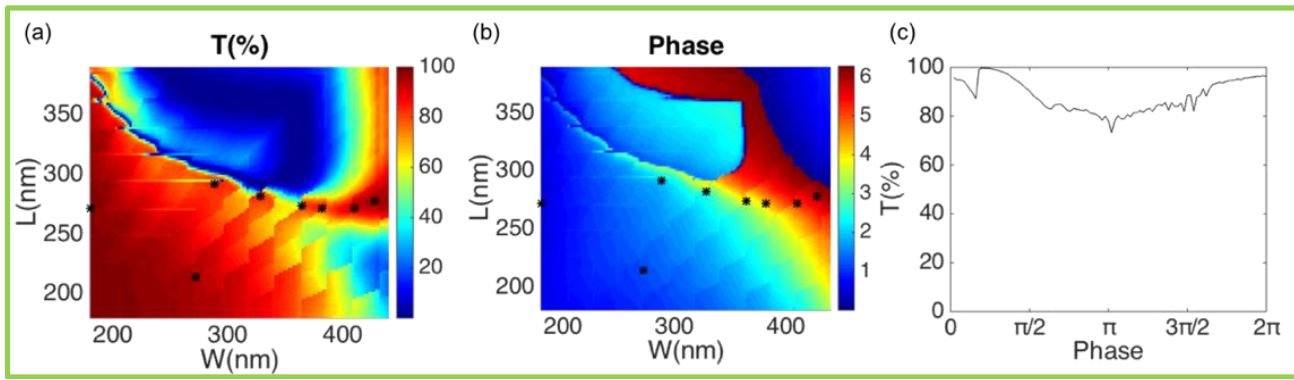

Figure 2. Design of meta-atoms. (a) Transmittance for nanoblocks on silica substrate as function of nanoblocks' width $\mathrm{W}$ and length $\mathrm{L}$ with $\mathrm{H}=132 \mathrm{~nm}$ and $\mathrm{P}=\max (\mathrm{W}, \mathrm{L})+90 \mathrm{~nm}$. (b) Corresponding phase variation of transmitted light through nanoblocks. (c) The highest transmittance achieved by the nanoblocks in the $0-2 \pi$ full-phase space. The period $P$ keeps constant $\max (\mathrm{W}, \mathrm{L})+90 \mathrm{~nm}$. The black star markers indicate the eight meta-atoms selected to construct metasurfaces.

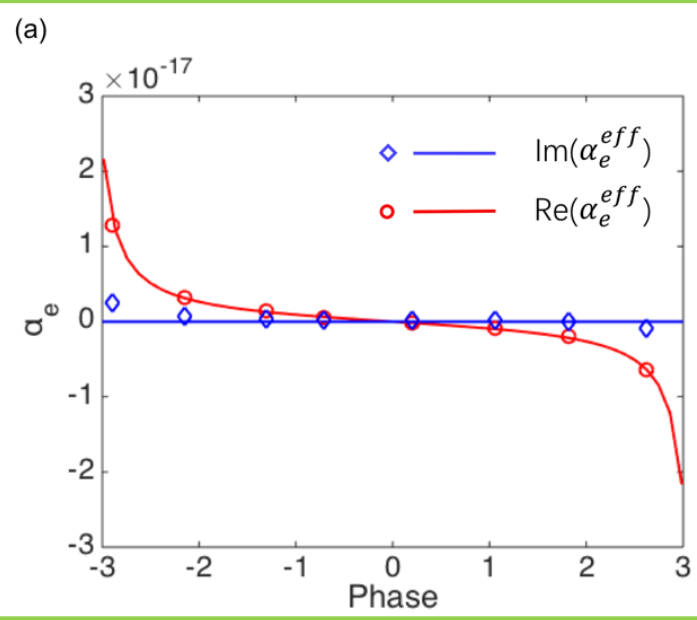

(b)

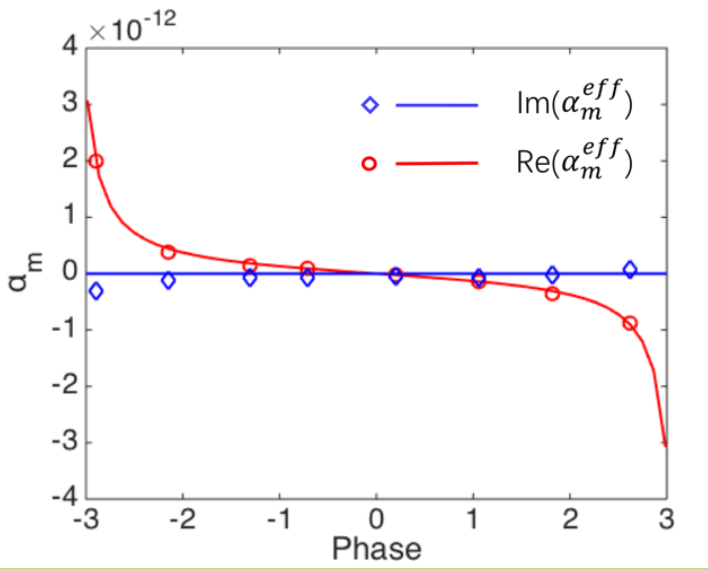

Figure 3. Effective (a) electric and (b) magnetic polarizabilities $\alpha_{e}^{\text {eff }} / \alpha_{m}^{\text {eff }}$ of the selected eight meta-atoms. The solid line is the ideal electromagnetic polarizabilities of Huygens meta-atoms.

\section{Metasurface Construction and Simulation}

Since the phase distribution function of the metasurface can be independently controlled by every single nanostructure in each microscopic region, independent phase manipulation can be performed on each point constituting the wavefront of the target beam. Therefore, we can design arbitrary phase functions based on the selected meta-atoms. We built a beam deflector (Figure 4) and simulated its performance. The phase function of abnormal refraction is $\varphi(x)=k_{x} \times x$, where $k_{x}$ is the phase 
gradient of the metasurface. The simulation results are shown in Figure $4 b$, which is the phase distribution on the $\mathrm{x}-\mathrm{z}$ plane. The incident plane light is deflected. The theoretical angle of deflection $\theta=\arcsin [(d \varphi / d l) /(2 \pi n / \lambda)] \approx 9.5^{\circ}$ (where, $\lambda$ is the wavelength of light in free space, and $d \varphi / d l$ is the phase gradient in the $x$-direction), which is consistent with the simulation results. The transmitted light conversion efficiency of the sample reaches $80 \%$. The transmission comparison between non-periodic and periodic arrays of meta-atoms is shown in Figure A1.

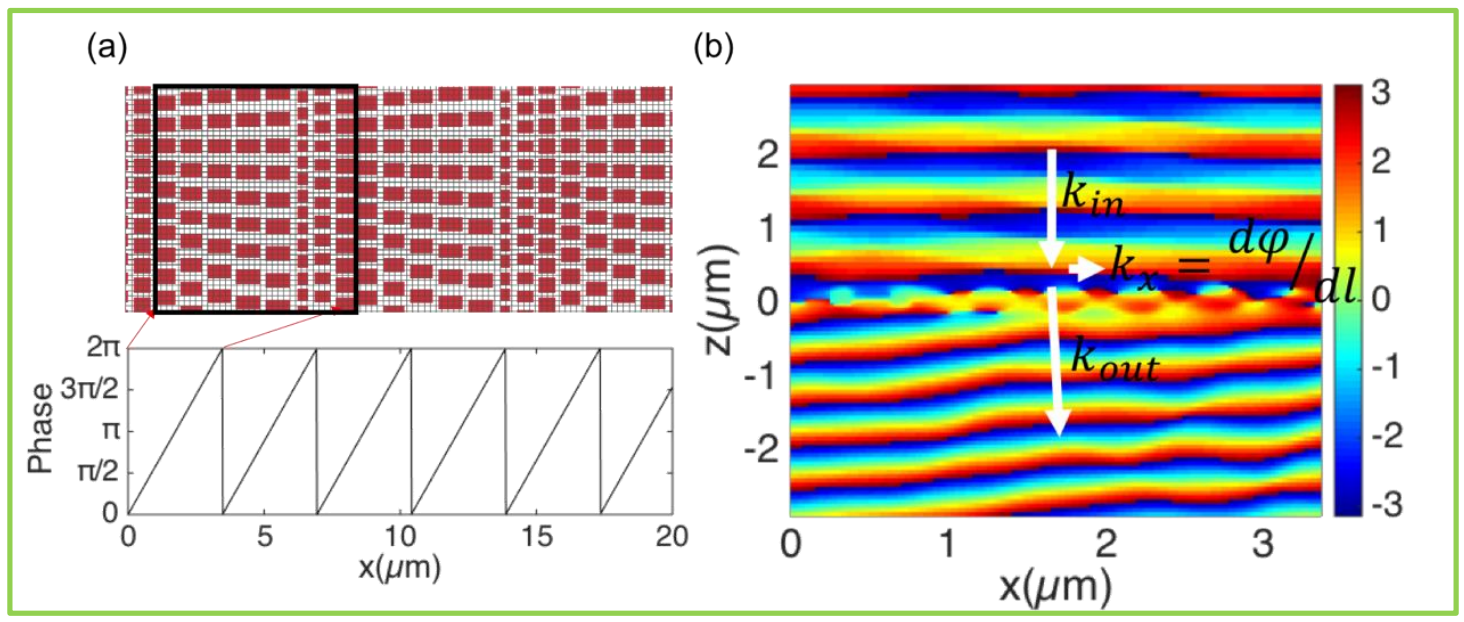

Figure 4. Metasurfaces of grating. (a) Top: schematics of grating composed of the eight meta-atoms in Figure 3, having the digital phase shift with $\pi / 4$ increments covering the $2 \pi$ range. Bottom: phase distribution function of the metasurface. (b) Phase distribution when a plane wave passes through the metasurface, showing deflection with angle $9.5^{\circ}$.

Furthermore, according to the phase function of the two-dimensional lens:

$$
\varphi(r, \lambda)=-\frac{2 \pi}{\lambda}\left(\sqrt{r^{2}+f^{2}}-f\right)+\varphi_{0}(\lambda)
$$

where $f$ is the focal length, $\lambda$ is the wavelength, and $\varphi_{0}(\lambda)$ is an arbitrary phase. The corresponding phase distribution is shown in Figure 5a. Based on the selected meta-atoms, a plane lens was constructed with focal length $\mathrm{f}=120 \mu \mathrm{m}$, aperture $\mathrm{D}=150 \mu \mathrm{m}$, as in Figure $5 \mathrm{~b}, \mathrm{c}$. The plane light is focused at the focal plane position, as shown in Figure 5d,e. Figure 5d shows the power distribution of the focusing spot on the $x-z$ plane, and the focal spot appears at the position of $z=115.1 \mu \mathrm{m}$. Figure 5e shows the power distribution of the focusing spot in the $x-y$ plane, i.e., the focal plane. The upper part of Figure $5 \mathrm{f}$ is the $\mathrm{x}-\mathrm{y}$ plane power distribution. The lower part is the power distribution with $y=0$, and the half peak full width (FWHM, $768 \mathrm{~nm}$ ) of the focal spot reaches the sub-wavelength magnitude. Compared to the metasurface based on periodic meta-atoms, our structure effectively avoids diffraction and interference. Furthermore, due to the sub-wavelength scale, the scattering of light is also reduced. Therefore, it achieved a focusing conversion efficiency (the power in the focused light spot (with diameter $3 \times$ FWHM divided by the total power on the focal plane) of $99.3 \%$ for all the transmitted light. It is worth noting that the focal plane position is at $\mathrm{z}=115.1 \mu \mathrm{m}$, which is slightly different from the designed focal length $(120 \mu \mathrm{m})$, which may be related to the position of the main plane of the lens. On the other hand, it may also be related to the height of the meta-atoms. 


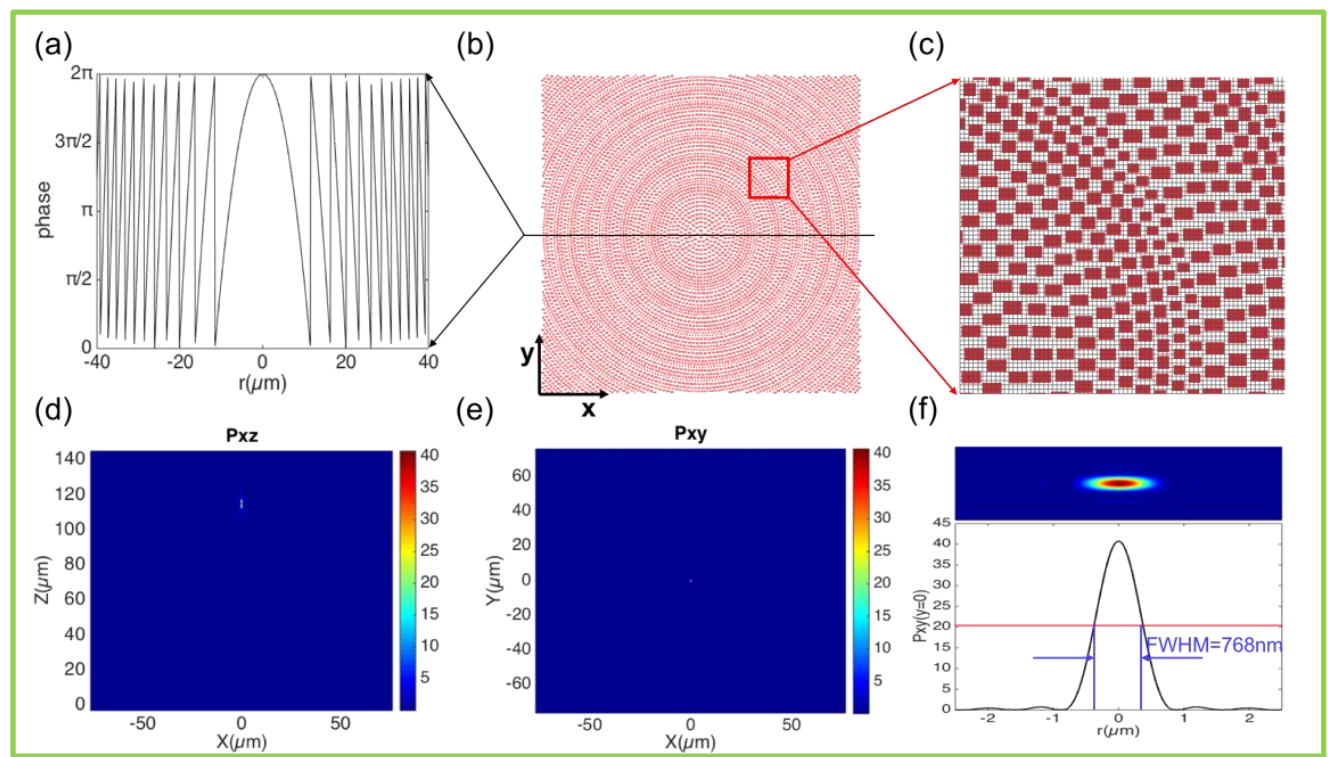

Figure 5. Metasurfaces of parabolic lens. (a) Phase-distribution function of the metalens in radial direction. (b) Top view of metalens composed of the eight meta-atoms in Figure 3, introducing the digital phase shift with $\pi / 4$ increments covering the $2 \pi$ range. (c) Partial enlarged details of the metasurface, which is marked by the red box in (b). (d,e) Power distributions of the focusing spot in the $\mathrm{x}-\mathrm{z}(\mathrm{y}=0 \mathrm{~nm})$ and $\mathrm{x}-\mathrm{y}(\mathrm{z}=115.1 \mu \mathrm{m})$ planes, respectively. (f) Full width at half maximum (FWHM) of the focusing spot.

When changing the focus length of the meta-lens, i.e., $f=60 \mu \mathrm{m}(\mathrm{D}=100 \mu \mathrm{m}), \mathrm{f}=80 \mu \mathrm{m}$ $(\mathrm{D}=100 \mu \mathrm{m})$, and $\mathrm{f}=120 \mu \mathrm{m}(\mathrm{D}=150 \mu \mathrm{m})$, the corresponding focusing efficiency increases, as shown in Figure 6. A larger focal length $\mathrm{f}$ means a smaller phase gradient of the meta-lens, and therefore the phase function digitalization of lens has higher accuracy spatially based on the eight selected meta-atoms. The F-numbers of the three meta-lens are $0.6,0.8$, and 0.8 , respectively. Therefore, the latter two lenses have similar FWHM values, $804 \mathrm{~nm}$ and $768 \mathrm{~nm}$, bigger than the first one at $660 \mathrm{~nm}$. Moreover, all the FWHM values are smaller than the wavelength of $830 \mathrm{~nm}$.

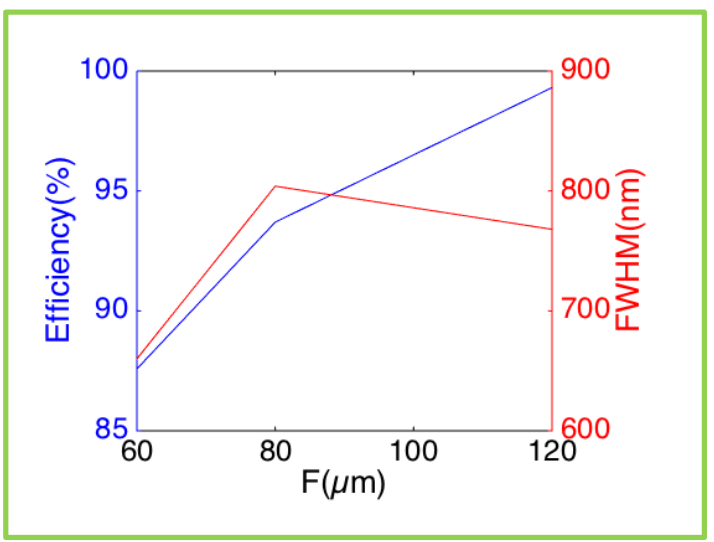

Figure 6. Focusing efficiency and FWHM of focus spot as function of the meta-less' focal length $\mathrm{f}$.

\section{Conclusions}

To sum up, based on aperiodic Si nanoarrays, metasurfaces with $2 \pi$ phase control are investigated. An abnormal refraction device with a deflection angle $9.5^{\circ}$ is realized, and the conversion efficiency of transmitted light is up to $80 \%$. Based on the phase function of the plane lens, a two-dimensional meta-lens is designed to achieve the perfect focusing with a focal length of $115.1 \mu \mathrm{m}$, an F-number of 
0.8 , a focal spot size of $768 \mathrm{~nm}$, and the focusing conversion efficiency of the transmitted light reaches $99.3 \%$. Most notably, we use aperiodic meta-atomic structures that greatly reduce the diffraction and interference effects of the source at the focal plane. Similarly, we can design corresponding optical metasurface devices according to any phase function. By combining with the current mature CMOS processing technology, large-scale industrial production of metasurface devices can be realized at a low cost, which can be widely used in industrial, military, and other fields.

Author Contributions: Conceptualization, S.S. and F.T.; methodology, Z.Z. and W.Z.; software, S.S. and F.T.; validation, S.S. and F.T.; formal analysis, F.T. and X.Y.; investigation, Q.L., H.L., J.W., Y.W., and J.C.; resources, X.Y., Z.Z., and W.Z.; data curation, S.S. and F.T.; writing-original draft preparation, S.S.; writing-review and editing, S.S., F.T., X.Y., Z.Z., and W.Z.; funding acquisition, Z.Z. and Y.Y. All authors have read and agree to the published version of the manuscript.

Funding: This research was funded by National Natural Science Foundation of China (grant number: 11404052, 11474048, 11874102, 61705204, 61705206), China Postdoctoral Science Foundation (grant number: 2019M653486), Sichuan Province Science and Technology Support Program (grant number: 2019YJ0187), and Innovation and Development Foundation of China Academy of Engineering Physics (grant number: CX20200021).

Acknowledgments: We are grateful to all the authors for their contributions to this article.

Conflicts of Interest: The authors declare no conflicts of interest.

\section{Appendix A}

The transmission comparison between non-periodic and periodic arrays of meta-atoms is shown in Figure A1. We can design more parameters without being limited by periodicity. As shown Figure A1a, compared with the periodic meta-atoms, the transmittance of aperiodic structures is higher. Moreover, for the far-field radiation intensity of the grating, compared with the periodic meta-atoms, the first-order diffraction order efficiency of the metasurfaces composed of aperiodic meta-atoms is higher, and the efficiency of the other orders is lower, which shown the advantage of the aperiodic meta-atoms, as shown Figure A1b.

(a)

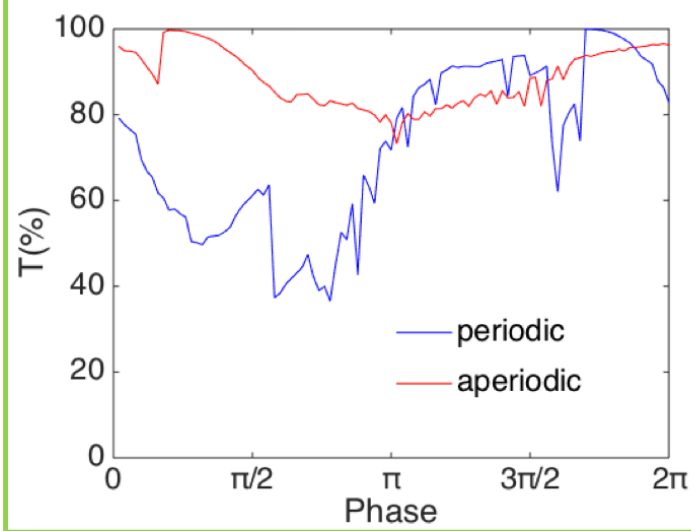

(b)

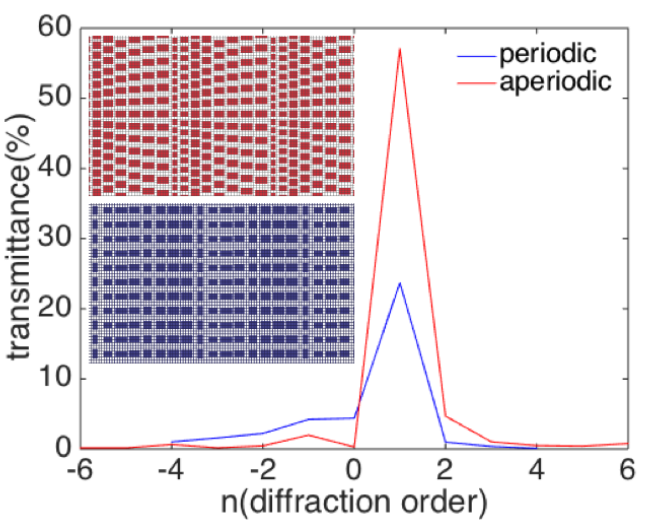

Figure A1. (a)The highest transmittance achieved by the comparison of periodic (blue) and aperiodic (red) nanoblocks in the 0-2 $\pi$ full-phase space, which shows that aperiodic significantly improves the corresponding efficiency. (b) Contrast diagram of far-field radiation intensity. The inserted red image is the grating composed of aperiodic meta-atoms, and the blue image is the grating composed of periodic meta-atoms.

The transmittance and phase of the selected meta-atoms with size errors are shown in Figure A2. The red curves with stars show the ideal case in which the selected meta-atoms have the sizes in Figure 2. If the fabrication has an error of $\pm 4 \mathrm{~nm}$, the transmittance and phase are shown by the blue and green curves. The transmittance of meta-atoms numbered 1,2, 7, and 8 stays almost the same 
while the others show a drop of $20 \%$. When the error increases to $-6 \mathrm{~nm}$, the transmittance decreases to $25 \%$. However, the error of $+6 \mathrm{~nm}$ introduces the transmittance change is similar to the error case of $\pm 4 \mathrm{~nm}$. In terms of phase, a bigger error brings a bigger offset from the desirable phase. The positive error makes the phase bigger than the desired value while the negative error brings a phase smaller than the desired value.

\section{(a)}

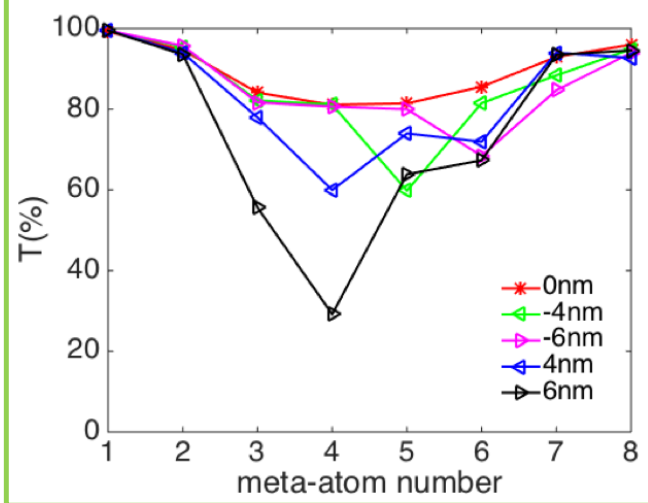

(b)

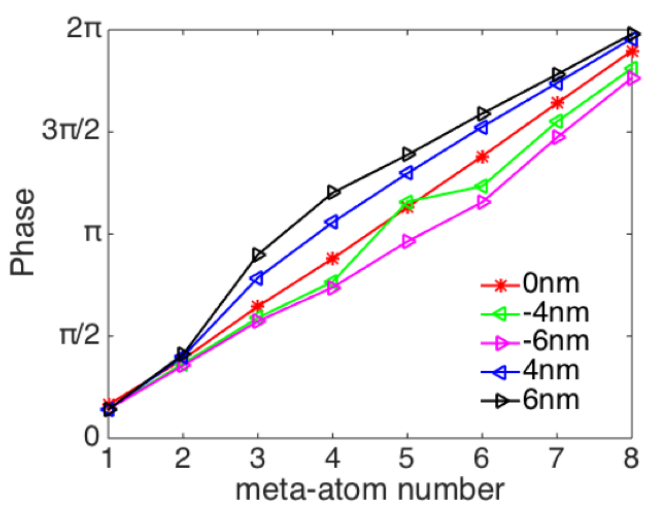

Figure A2. Transmittance and phase of the selected meta-atoms with size errors.

We calculated our $Q$ factors and dephasing times $[43,44]$ of all the electromagnetic resonances of the Si nanoblocks. As shown in Figure A3a, the Q factors and dephasing time of the electromagnetic resonances of the Si nanoblock in Figure 1 are calculated. Figure A3b,c shows the $Q$ factors and dephasing time of the electromagnetic resonances of the eight selected meta-atoms in Figure 2.

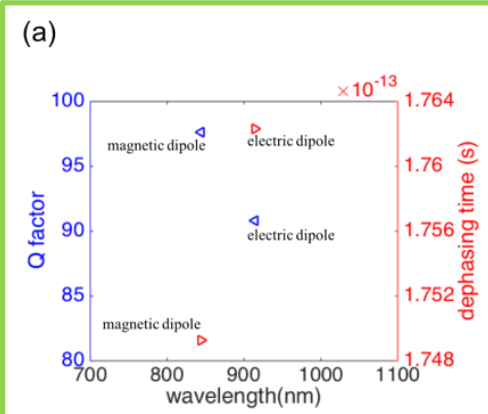

\section{(b)}

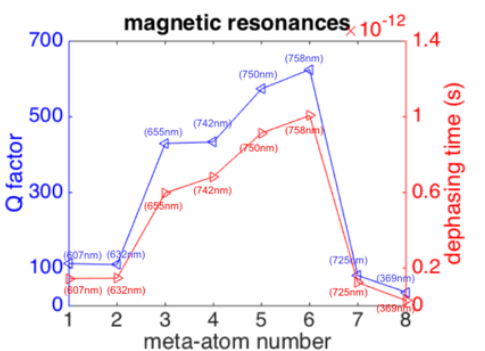

(c)

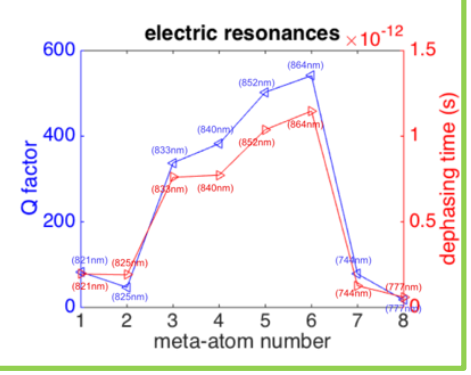

Figure A3. Q factors (blue marks) and dephasing times (red marks) of: (a) the electric dipole and magnetic dipole in Figure 1; (b) the magnetic resonances; and (c) electric resonances of the eight selected meta-atoms that are marked by stars in Figure 2.

\section{References}

1. Lin, R.J.; Su, V.C.; Wang, S.; Chen, M.K.; Chung, T.L.; Chen, Y.H.; Kuo, H.Y.; Chen, J.W.; Chen, J.; Huang, Y.T.; et al. Achromatic Metalens Array for Full-Colour Light-Field Imaging. Nat. Nanotechnol. 2019, 14, $227-231$. [CrossRef] [PubMed]

2. Khorasaninejad, M.; Chen, W.T.; Devlin, R.C.; Oh, J.; Zhu, A.Y.; Capasso, F. Metalenses at Visible Wavelengths: Diffraction-Limited Focusing and Subwavelength Resolution Imaging. Science 2016, 352, 1190-1194. [CrossRef] [PubMed]

3. Pillai, S.; Catchpole, K.R.; Trupke, T.; Green, M.A. Surface Plasmon Enhanced Silicon Solar Cells. Br. J. Appl. Phys. 2007, 101, 093105. [CrossRef]

4. Deng, Z.L.; Zhang, S.; Wang, G.P. A Facile Grating Approach Towards Broadband, Wide-Angle and High-Efficiency Holographic Metasurfaces. Nanoscale 2016, 8, 1588-1594. [CrossRef] [PubMed] 
5. Liu, D.; Wu, J.; Guo, S. The Generation of Three-Dimensional Curved Beams Based on Holographic Metasurface. Opt. Express 2018, 26, 22348-22355. [CrossRef] [PubMed]

6. Zhang, Z.H.; Shang, S.H.; Li, M.N.; Wu, S.Y.; Zhu, Q.S.; Wu, M.H.; Teng, B.H. Fdtd Simulation: Refractive Index and Single-Object Sensing Using a Whispering-Gallery-Modes Microring Resonator. J. Lightw. Technol. 2018, 36, 1775-1781. [CrossRef]

7. Yang, Y.; Zhao, Q.; Liu, L.; Liu, Y.; Rosales-Guzmán, C.; Qiu, C.W. Manipulation of Orbital-Angular-Momentum Spectrum Using Pinhole Plates. Phys. Rev. Appl. 2019, 12, 064007. [CrossRef]

8. Liu, L.; Zhang, X.; Kenney, M.; Su, X.; Xu, N.; Ouyang, C.; Shi, Y.; Han, J.; Zhang, W.; Zhang, S. Broadband Metasurfaces with Simultaneous Control of Phase and Amplitude. Adv. Mat. 2014, 26, 5031-5036. [CrossRef]

9. Shao, T.; Sun, L.; Li, W.; Zhou, X.; Wang, F.; Huang, J.; Ye, X.; Yang, L.; Zheng, W. Understanding the Role of Fluorine-Containing Plasma on Optical Properties of Fused Silica Optics During the Combined Process of Rie and Dce. Opt. Express 2019, 27, 23307-23320. [CrossRef]

10. Sun, L.; Liu, H.; Huang, J.; Ye, X.; Xia, H.; Li, Q.; Jiang, X.; Wu, W.; Yang, L.; Zheng, W. Reaction Ion Etching Process for Improving Laser Damage Resistance of Fused Silica Optical Surface. Opt. Express 2016, 24, 199-211. [CrossRef]

11. Luo, J.; Yang, Y.; Yao, Z.; Lu, W.; Hou, B.; Hang, Z.H.; Chan, C.T.; Lai, Y. Ultratransparent Media and Transformation Optics with Shifted Spatial Dispersions. Phys. Rev. Lett. 2016, 117, 223901. [CrossRef] [PubMed]

12. Zhu, B.O.; Zhao, J.; Feng, Y. Active Impedance Metasurface with Full $360^{\circ}$ Reflection Phase Tuning. Sci. Rep. 2013, 3, 3059. [CrossRef] [PubMed]

13. Arbabi, A.; Horie, Y.; Bagheri, M.; Faraon, A. Dielectric Metasurfaces for Complete Control of Phase and Polarization with Subwavelength Spatial Resolution and High Transmission. Nat. Nanotechnol. 2015, 10, 937. [CrossRef] [PubMed]

14. Yu, Y.F.; Zhu, A.Y.; Paniagua-Domínguez, R.; Fu, Y.H.; Luk'yanchuk, B.; Kuznetsov, A.I. High-Transmission Dielectric Metasurface with $2 \pi$ Phase Control at Visible Wavelengths. Laser $\mathcal{E}$ Photonics Rev. 2015, 9, 412-418.

15. Pun, E.Y.B.; Wong, P.W.H.; Li, G.; Chen, S.; Pholchai, N.; Reineke, B.; Cheah, K.W.; Zentgraf, T.; Zhang, S. Nonlinear Metasurface for Continuous Phase Control. In Proceedings of the 2016 IEEE MTT-S International Conference on Numerical Electromagnetic and Multiphysics Modeling and Optimization (NEMO), Beijing, China, 27-29 July 2016.

16. Wang, W.; Guo, C.; Tang, J.; Zhao, Z.; Wang, J.; Sun, J.; Shen, F.; Guo, K.; Guo, Z. High-Efficiency and Broadband near-Infrared Bi-Functional Metasurface Based on Rotary Different-Size Silicon Nanobricks. Nanomaterials 2019, 9, 1744. [CrossRef]

17. Yang, Y.; Wang, W.; Moitra, P.; Kravchenko, I.I.; Briggs, D.P.; Valentine, J. Dielectric Meta-Reflectarray for Broadband Linear Polarization Conversion and Optical Vortex Generation. Nano Lett. 2014, 14, 1394-1399. [CrossRef]

18. Chen, W.T.; Yang, K.Y.; Wang, C.M.; Huang, Y.W.; Sun, G.; Chiang, I.D.; Liao, C.Y.; Hsu, W.L.; Lin, H.T.; Sun, S.; et al. High-Efficiency Broadband Meta-Hologram with Polarization-Controlled Dual Images. Nano Lett. 2014, 14, 25-30. [CrossRef]

19. Shibanuma, T.; Maier, S.A.; Albella, P. Polarization Control of High Transmission/Reflection Switching by All-Dielectric Metasurfaces. Appl. Phys. Lett. 2018, 112, 063103. [CrossRef]

20. Huang, Y.W.; Chen, W.T.; Tsai, W.Y.; Wu, P.C.; Wang, C.M.; Sun, G.; Tsai, D.P. Aluminum Plasmonic Multicolor Meta-Hologram. Nano Lett. 2015, 15, 3122-3127. [CrossRef]

21. Pors, A.; Nielsen, M.G.; Eriksen, R.L.; Bozhevolnyi, S.I. Bozhevolnyi. Broadband Focusing Flat Mirrors Based on Plasmonic Gradient Metasurfaces. Nano Lett. 2013, 13, 829-834. [CrossRef]

22. Peyskens, F.; Subramanian, A.Z.; Neutens, P.; Dhakal, A.; Van Dorpe, P.; Le Thomas, N.; Baets, R. Bright and Dark Plasmon Resonances of Nanoplasmonic Antennas Evanescently Coupled with a Silicon Nitride Waveguide. Opt. Express 2015, 23, 3088-3101. [CrossRef] [PubMed]

23. Wang, W.; Zhao, Z.; Guo, C.; Guo, K.; Guo, Z. Spin-Selected Dual-Wavelength Plasmonic Metalenses. Nanomaterials 2019, 9, 761. [CrossRef] [PubMed]

24. Miscuglio, M.; Borys, N.J.; Spirito, D.; Martín-García, B.; Zaccaria, R.P.; Weber-Bargioni, A.; Schuck, P.J.; Krahne, R. Planar Aperiodic Arrays as Metasurfaces for Optical near-Field Patterning. ACS Nano 2019, 13, 5646-5654. [CrossRef] [PubMed] 
25. Chen, S.; Chen, Z.; Liu, J.; Cheng, J.; Zhou, Y.; Xiao, L.; Chen, K. Ultra-Narrow Band Mid-Infrared Perfect Absorber Based on Hybrid Dielectric Metasurface. Nanomaterials 2019, 9, 1350. [CrossRef]

26. Zhang, J.; Liu, W.; Zhu, Z.; Yuan, X.; Qin, S. Strong Field Enhancement and Light-Matter Interactions with All-Dielectric Metamaterials Based on Split Bar Resonators. Opt. Express 2014, 22, 30889-30898. [CrossRef]

27. Ahmadivand, A.; Karabiyik, M.; Pala, N. Inducing Multiple Fano Resonant Modes in Split Concentric Nanoring Resonator Dimers for Ultraprecise Sensing. J. Opt. 2015, 17, 085104. [CrossRef]

28. Semouchkina, E.; Duan, R.; Semouchkin, G.; Pandey, R. Sensing Based on Fano-Type Resonance Response of All-Dielectric Metamaterials. Sensors 2015, 15, 9344-9359. [CrossRef]

29. Decker, M.; Staude, I.; Falkner, M.; Dominguez, J.; Neshev, D.N.; Brener, I.; Pertsch, T.; Kivshar, Y.S. High-Efficiency Dielectric Huygens' Surfaces. Adv. Opt. Mat. 2015, 3, 813-820. [CrossRef]

30. Shalaev, M.I.; Sun, J.; Tsukernik, A.; Pandey, A.; Nikolskiy, K.; Litchinitser, N.M. High-Efficiency All-Dielectric Metasurfaces for Ultracompact Beam Manipulation in Transmission Mode. Nano Lett. 2015, 15, 6261-6266. [CrossRef]

31. Zhao, W.; Jiang, H.; Liu, B.; Song, J.; Jiang, Y.; Tang, C.; Li, J. Dielectric Huygens' Metasurface for High-Efficiency Hologram Operating in Transmission Mode. Sci. Rep. 2016, 6, 30613. [CrossRef]

32. Chen, M.; Kim, M.; Wong, A.M.; Eleftheriades, G.V. Eleftheriades. Huygens' Metasurfaces from Microwaves to Optics a Review. Nanophotonics 2018, 7, 25. [CrossRef]

33. Kim, M.; Wong, A.M.; Eleftheriades, G.V. Optical Huygens' Metasurfaces with Independent Control of the Magnitude and Phase of the Local Reflection Coefficients. Phys. Rev. X 2014, 4, 041042. [CrossRef]

34. Sun, Z.; Sima, B.; Zhao, J.; Feng, Y. Electromagnetic Polarization Conversion Based on Huygens' Metasurfaces with Coupled Electric and Magnetic Resonances. Opt. Express 2019, 27, 11006-11017. [CrossRef]

35. Li, X.; Chen, G.; Yang, L.; Jin, Z.; Liu, J. Multifunctional Au-Coated Tio2 Nanotube Arrays as Recyclable Sers Substrates for Multifold Organic Pollutants Detection. Adv. Funct. Mater. 2010, 20, 2815-2824. [CrossRef]

36. Hsiao, H.H.; Chu, C.H.; Tsai, D.P. Fundamentals and Applications of Metasurfaces. Small Methods 2017, 1, 1600064. [CrossRef]

37. Arbabi, A.; Briggs, R.M.; Horie, Y.; Bagheri, M.; Faraon, A. Efficient Dielectric Metasurface Collimating Lenses for Mid-Infrared Quantum Cascade Lasers. Opt. Express 2015, 23, 33310-33317. [CrossRef]

38. Arbabi, E.; Arbabi, A.; Kamali, S.M.; Horie, Y.; Faraji-Dana, M.; Faraon, A. Mems-Tunable Dielectric Metasurface Lens. Nat. Commun. 2018, 9, 812. [CrossRef]

39. Colburn, S.; Zhan, A.; Majumdar, A. Tunable Metasurfaces Via Subwavelength Phase Shifters with Uniform Amplitude. Sci. Rep. 2017, 7, 40174. [CrossRef]

40. Stadler, I.; Lanzafame, R.J.; Evans, R.; Narayan, V.; Dailey, B.; Buehner, N.; Naim, J.O. 830-Nm Irradiation Increases the Wound Tensile Strength in a Diabetic Murine Model. Lasers Surg. Med. Off. J. Am. Soc. Laser Med. Surg. 2001, 28, 220-226. [CrossRef]

41. Hagiwara, S.; Iwasaka, H.; Okuda, K.; Noguchi, T. Gaalas (830 Nm) Low-Level Laser Enhances Peripheral Endogenous Opioid Analgesia in Rats. Lasers Surg. Med. 2007, 39, 797-802. [CrossRef]

42. Chow, R.T.; Heller, G.Z.; Barnsley, L. The Effect of 300 Mw, 830 Nm Laser on Chronic Neck Pain: A Double-Blind, Randomized, Placebo-Controlled Study. Pain 2006, 124, 201-210. [CrossRef] [PubMed]

43. Ahmadivand, A.; Gerislioglu, B.; Ramezani, Z. Gated Graphene Island-Enabled Tunable Charge Transfer Plasmon Terahertz Metamodulator. Nanoscale 2019, 11, 8091-8095. [CrossRef] [PubMed]

44. Sönnichsen, C.; Franzl, T.; Wilk, T.; Von Plessen, G.; Feldmann, J. Plasmon Resonances in Large Noble-Metal Clusters. New J. Phys. 2002, 4, 93. [CrossRef]

(C) 2020 by the authors. Licensee MDPI, Basel, Switzerland. This article is an open access article distributed under the terms and conditions of the Creative Commons Attribution (CC BY) license (http://creativecommons.org/licenses/by/4.0/). 